Gerd-Helge Vogel

\title{
CARL JULIUS SENFF (1804-1832) - EIN KURZES KÜNSTLERLEBEN ALS ARCHITEKT, ZEICHNER UND GRAFIKER
}

Unter den hoffnungsversprechenden Schülern von Carl August Senff befand sich sein ältester Sohn Carl Julius Senff - ein mit vielseitigen Interessen versehener, begabter Mensch, der sich der Architektur widmen wollte und aus dem sich ein Künstler mit einem auffallenden Schaffen entwickelte. ${ }^{1}$ In diesem Beitrag werden das Leben des Künstlers und sein Werk betrachtet, welches bis zur letzten Zeit nur spärlich untersucht wurde.

Einer der frühesten Versuche geht bis ins Jahr 1845 zurück und stammt aus der Feder von Georg Kaspar Nagler, der bereits zwölf Jahre nach dem Tode des früh verstorbenen Künstlers lapidar im 15. Bande seines Neuen allgemeinen Künstler-Lexikons die wesentlichsten Fakten vermerkte: „[...] Zeichner und Maler, arbeitete längere Zeit in Berlin und zu Prag. Seine Werke bestehen in landschaftlichen und architektonischen Darstellungen. Darunter ist ein solches über den Dom in Prag, unter dem Titel: die Domkirche zu St. Veit in Prag, gezeichnet und

1 Über Carl Julius Senff hat der Verfasser eine Übersicht veröffentlicht: Gerd-Helge Vogel, „Carl Julius Senff - üks lühike kunstniku elu“, Eesti kunsti ajalugu 3, 1770-1840, hrsg. von Juhan Maiste (Tallinn: Eesti Kunstiakadeemia, 2017), 222-225. 


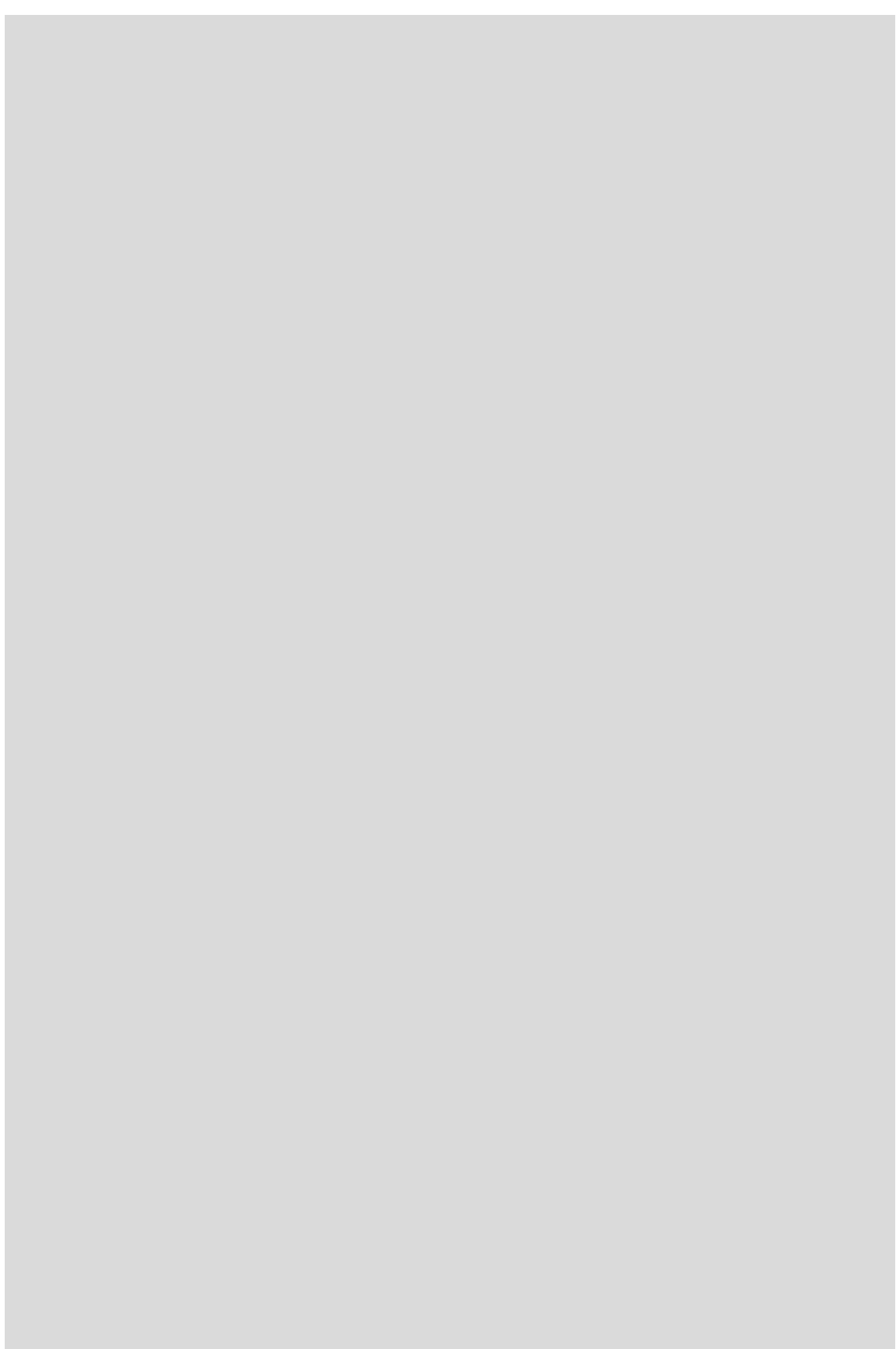

Abb. 1. Carl Julius Senff, Südwest Ansicht der Veitskirche zu Prag. Aquatinta, 1831. Estnisches Kunstmuseum. geätzt von C. J. Senff. Mit 10 K. Berlin 1831, gr. fol.“2 (Abb. 1) Über diese kurzen Informationen geht selbst Thieme/Beckers Künstlerlexikon aus der ersten Hälfte des 20. Jahrhunderts kaum hinaus, denn es vermerkt nur zusätzlich, dass er 1830 in Jena zum Dr. phil. promovierte, in Prag und Wien Architektur studierte und gemeinsam mit seinem Onkel Adolf Italien bereiste. ${ }^{3}$ Schätzbar ist dabei vor allem der Hinweis auf den Eintrag von Wilhelm Neumann in dessen Lexikon baltischer Künstler aus dem Jahre 1908, wo zu erfahren ist, dass er „von 1823-27 an der Universität Dorpat (studierte) und [...] als Student ein Lehrbuch der Perspektive für Landschaftsmaler zum Selbststudium (verfasste und) seine preisgekrönte Arbeit „Systematische Darstellung der Hauptsätze der Geometrie im Raume“, [...] auf Kosten der Universität gedruckt (wurde)“. Diese wenigen Angaben werden durch den Hinweis ergänzt dass er auf der Rückreise seines Italienaufenthalts erkrankte und bewusstlos nach Mailand gebracht wurde, wo er verstarb, weshalb er an der Universität Dorpat (Tartu) die Professur für Architektur nicht antreten konnte, für die er designiert war. ${ }^{4}$ Eine kurze Liste von vier Werkgruppen - Radierungen, Lithographien, Aquatinten und eine Illustrationsserie - vervollständigt schließlich diese Biografie, mit der faktisch bereits alles Wissenswerte über den hoffnungsvollen, früh vollendeten Künstler zusammengefasst wurde, ${ }^{5}$ denn spätere Erwähnungen und Darstellungen über Carl Julius Senff gehen über diese Notizen eigentlich nicht hinaus. ${ }^{6}$ Doch zum Glück existieren im Estnischen Historischen Archiv (Eesti Ajalooarhiiv) in Tartu zwe umfangreiche Akten über Carl Julius Senff ${ }^{7}$, aus denen sich noch manch zusätzliches wissenswertes Detail zu Leben und Werk dieses interessanten deutschbaltischen Künstlers erschließt. Zusammen mit dem erhaltenen Konvolut von Zeichnungen und Graphiken seiner

2 Georg K. Nagler, Neues allgemeines Künstler-Lexicon oder Nachrichten von dem Leben und den Werken der Maler, Bildhauer, Baumeister, Kupferstecher, Formschneider, Lithographen, Zeichner, Medailleure, Elfenbeinarbeiter, etc, Bd. XV (München: Fleischmann, 1845), 272.

3 Allgemeines Lexikon der bildenden Künstler von der Antike bis zur Gegenwart begründet von Ulrich Thieme und Felix Becker, Bd. I-XXXVII, hrsg. von Hans Vollmer (Leipzig: Seemann, 907-1950), hier Bd. XXX, 497.

4 Wilhelm Neumann, Lexikon baltischer Künstler (Riga: Jonck \& Poliewsky, 1908), 150

5 Ibidem, $150-151$.

6 Günter Krüger, „Die Zeichenschule der Universität Dorpat“, Die Zeichenschule der Universität Dorpat 1803-1891, T. I. Unter der Leitung von Karl August Senff von 1803-1838 (Husum: Husum Druck- und Verlagsgesellschaft, 1993), 69.

7 Estnisches Historisches Archiv [Eesti Ajalooarhiiv, EAA], 402-2-23084-23085. 
Hand im Estnischen Kunstmuseum Tallinn lässt sich so sein kurzer Entwicklungsweg verfolgen, wichtige Aspekte der Studienzeit beleuchten und seine Reise nach Deutschland und Italien teilweise rekonstruieren.

\section{UNBEKANNTE ARCHIVALISCHE INFORMATIONEN}

Über die Kindheit von Julius Senff wird nichts in den Akten vermeldet, doch ist bekannt, dass sein Vater, der Kupferstecher und Universitätszeichenmeister, den ältesten Sohn schon früh in die Geheimnisse seiner Kunst einwies, indem er ihn im Zeichnen und in druckgrafischen Techniken unterrichtete, so dass Carl August Senff seinen Sohn als Gehilfen bei der Erledigung seiner zahlreichen Aufgaben mit einspannen konnte. Wie eng sich diese Zusammenarbeit gestaltete, wird aus den ältesten überlieferten Dokumenten ersichtlich, etwa dem Schreiben des Professors Carl August Senff an seine Magnifizenz, den Staatsrat, Ritter und Rektor der Universität Dorpat, Johann Philipp Gustav von Ewers (1779-1830) mit der "gehorsamsten Bitte“ vom 28. Juni 1823, die folgenden Wortlaut hat: „Mehrere hiesige Studierende hatten mich gebeten, das Portrait des Herrn Professor Erdmann zu malen, und ihm zu Ehre und Angedenken auf Stein zu zeichnen, damit jeder seiner bisherigen Schüler einen Abdruck seines Portraits erhalten könnte. Das Gemälde ist vollendet, aber die Zeichnung auf Stein muß ich in Petersburg machen, weil mir hier die nöthigen Steine und die ganze Vorzeichnung zum Steinabdruck mangeln. Da ich nicht im Stande bin schon jetzt zu bestimmen, wie lange ich mich zu Beendigung dieses Geschäftes in Petersburg verweilen muß, so ersuche ich Ew. Magnifizenz gehorsamst mir bey Einem Hochverordnetem Conseil einen Urlaub von 28 Tagen, von Ende der Ferien an gerechnet, auszuwirken, / Und zugleich auch meinen Sohne, dem ich zu meiner Hülfe mit nach Petersburg nehme, die Erlaubniß zu ertheilen, nicht mit mir zugleich zurückkehren zu dürfen ohne daß für ihn ein Nachtheil damit entsteht, wann er vielleicht einige Tage oder Wochen nach den gesetzlichen Termin zurückkehrt [...] ${ }^{\prime 8}{ }^{8}$ Dem „Extract aus dem Protocoll der Kaiserl. Univ. zu Dorpat d. d. 23. Aug. 1823“ ist zu entnehmen, dass dieser Bitte stattgegeben wurde, „[...] wobei H. Prorector referierte, daß der Urlaub im
Namen des Conseils vortheilht sey“. ${ }^{9}$ Carl Julius war seit dem 12. Januar 1823 an der Universität Dorpat als Student der Philosophie eingeschrieben ${ }^{10}$ und aus dem Attest seines Studienabschlusses vom 7. September 1827 wird ersichtlich, dass er in den „von ihm fleißig frequentierten Vorlesungen Special-Zeugnisse beygebracht (habe) über Mineralogie, allgemeine theoretische u. experimentale Chemie, Differentiiae- u. Integral-Rechnung, analytische Geometrie, hohe Mechanik, Psychologie, Logik, Grundsätze der Baukunst, Physik 1sten u. 2 Theil Politik, neueste Geschichte u. Geschichte der Russen [...]“.11 Anders als in heutiger Zeit, umfasste das Studium der Philosophie ein weites Spektrum auch von naturwissenschaftlichen Kenntnissen. Allerdings unterwarf sich Carl Julius bei seinem Abgang von der Universität zunächst keiner Prüfung, weshalb der Conseil es sich nicht erlaubte, über Senffs erworbene Kenntnisse ein Urteil zu fällen. Doch immerhin wurde ihm die „Allergnädigst verliehene Rangklasse bestätig[t und] seine sittliche Führung die stets musterhaft war, gebührt(e) ausgezeichnetes Lob“. ${ }^{12}$ Doch schon einige Tage später, am 8 . September 1827, stellte der "gewesene Studierende der Philosophie Julius Senff (ein Gesuch) um das vorschriftsmäßige Gradualexamen“13, das er am folgenden Tage nachmittags 3 Uhr bis abends 9 Uhr mündlich in Gegenwart der Professoren Egelhardt, Jäsche, Bartels, Ledebour, Perwostschikow, Parrot und Eschscholz ablegte, dem das schriftliche Examen am 10. September folgte. ${ }^{14}$ Beide Examen verliefen erfolgreich, denn die aus der Arithmetik, der Geometrie, der Algebra, der Trigonometrie, der Differentialund Integralrechnung, der Astronomie, der Mechanik, der Chemie, der Physik, der Botanik, der Logik und der russischen Sprache gestellten Fragen beantwortete der Prüfling „in solchem Maße zur Zufriedenheit Sr. Lehrer, daß sie ihm einstimmig den CanditatenGrad zuerkannten“. ${ }^{15}$

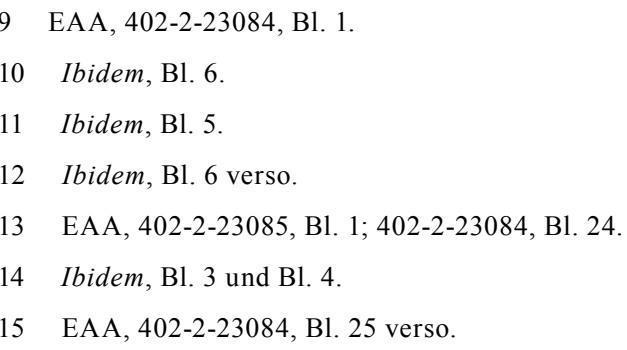


Zweifelsfrei war der Studiosus Senff während seiner vierjährigen Studienzeit in Dorpat durch seine zahlreichen außerordentlichen Leistungen aufgefallen und er hatte auf das Conseil eine beeindruckende Wirkung ausgeübt, denn mit dem von ihm verfassten Lehrbuch der Perspektive für Landschaftsmaler zum Selbststudium und der auf Universitätskosten gedruckten, preisgekrönten Arbeit „Systematische Darstellung der Hauptsätze der Geometrie im Raume ${ }^{\text {"16 }}$ erwarb sich der begabte Jüngling erste akademische Anerkennung, die es der Leitung der Universität wünschenswert erscheinen ließ, ihn für die spätere Übernahme wichtiger Aufgaben an der Alma mater Dorpatiensis nach Kräften zu fördern.

Doch zunächst ging der junge Carl Julius Senff nach Abschluss seiner Dorpater Studien zur weiteren Ausbildung nach Helsingfors (Helsinki), wo er beim dortigen Architekten - einem herausragenden Repräsentanten der Berliner Schule - Carl Ludwig Engel (1778-1840) "dem Bau-Wesen oblag", wie dem Protokoll des Conseils vom 9. Oktober $1828 \mathrm{zu}$ entnehmen ist. ${ }^{17}$ Für den jungen Senff gab es unter diesen, für die Baukunst günstigen Bedingungen unter dem Mentorat von C. L. Engel enorm viel zu lernen und so nimmt es nicht wunder, dass das Conseil der heimatlichen Universität in Tartu nach dem Ableben des ordentlichen Professors der Ökonomie, Technologie und bürgerlichen Baukunst, Johann Wilhelm Krause (1757-1828), „wegen der [...] Schwierigkeiten, die es geben würde, für die Architectur einen tüchtigen Mann zu finden, [...], de(n) Cand. Senff" als dessen Nachfolger für einem nur der Baukunst gewidmeten Lehrstuhl ins Auge zu fassen, weil er "sowohl Talent als Neigung habe (sich) ganz diesem Fache zu widmen" ${ }^{18}$ Ein dreijähriges Reisestipendium von jährlich 200 Dukaten sollte von der hohen Krone erbeten werden, um ihm die Möglichkeit zu schaffen, „sich im Auslande zweckmäßig ausbilden (zu) könne(n); unter der Bedingung, daß er nach Rückkehr bei dieser Univ. als außerord. Professor der Architectur auf 6 Jahre in Dienst trete, wenn ihn das Conseil dazu erwählt, oder wenn (er)

16 Diese von Neumann, Lexikon baltischer Künstler, 150, erwähnten Arbeiten Carl Julius Senffs ließen sich nicht mehr im Archiv nachweisen, wohl aber der Hinweis auf die schriftliche Abhandlung Systematische Darstellung der Hauptsätze der Geometrie im Raume, von Carl Julius Senff. Eine des Preises der silbernen Medaille und der Auszeichnung des Drucks auf Kosten der Universität am 12. Dezember 1827 würdig erkannte Preisschrift (Dorpat: J. C. Schünmann,

17 EAA, 402-2-23084, B1. 7.

18 Ibidem. nicht in demselben Fache auf ebenso lange Zeit einen anderen [...] Posten im Bau-Wesen übernehme ". ${ }^{19}$ Nachdem am 17. November 1828 „der Vater des gedachten Cand. Senff [...] dieser Behörde auf die deshalb an ihn ergangene Aufforderung die Versicherung ertheil(t)e, daß sein Sohn geneigt sey, solche Verpflichtung gegen die hohe Krone unter den angeführten Bedingungen zu übernehmen“20, konnte das Lehrprogramm für das Reisestipendium erarbeitet werden. So wurde am 30. Dezember 1828 „,verfügt, dem Herrn Cand. zu berichten, wo es nach der Meinung des Conseils dem Cand. Senff ein Aufenthalt in Berlin, München u. Paris zur Ausbildung für sein Fach am vortheilhaftesten sein würde, u. die UnterhaltungsGelder aus dem Fonds des Ministerii des öffentlichen Unterrichts zu verabfolgen wäre“. ${ }^{21}$ Schließlich erfolgte am 1. April 1829 die Verfügung des Conseils, dass „der außerordentliche Prof. Hofrath Senff Senior, in Kenntniß zu setzen (sei), mit dem Ersuchen, seinen Sohn anzuhalten, sich unverzüglich aus Helsingfors nach Dorpat zu [...] begeben, um seine Bestimmung anzutreten“. ${ }^{22}$ Inzwischen war die Planung der Auslandsaufenthalte neben Berlin, München und Paris auch um die Destination Italien, „das classische Land der Künstler" erweitert worden ${ }^{23}$ und am 11. Mai auf Allerhöchsten Befehl die jährliche Summe von 200 Dukaten zur Auszahlung gegen Quittung an den Cand. Senff angewiesen. ${ }^{24}$ Nachdem nach erfolgreichem Prüfungsabschluss am 8. Juni 1829 Carl Julius „die Würde u. die Rechte eines Candidaten der Philosophie“ verliehen worden waren, die in der damaligen Feudalhierarchie Russlands mit dem Erwerb des „Rang(es) der Zehnten Classe verbunden “25 waren, standen seinem Studienaufenthalt im Ausland keine Hindernisse mehr im Wege. So berichtet das Conseil am 20. Juni 1829 dem Rektor, dass "Carl Julius Senff in Folge der Allerhöchsten Genehmigung am heutigen Tage seine Reise ins Ausland angetreten hat ${ }^{2} .{ }^{26}$ Die für das Jahr

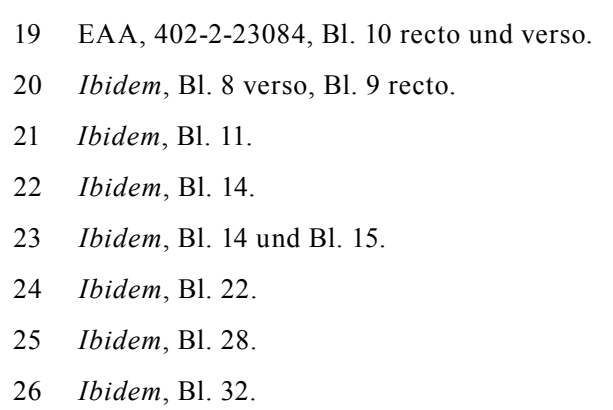




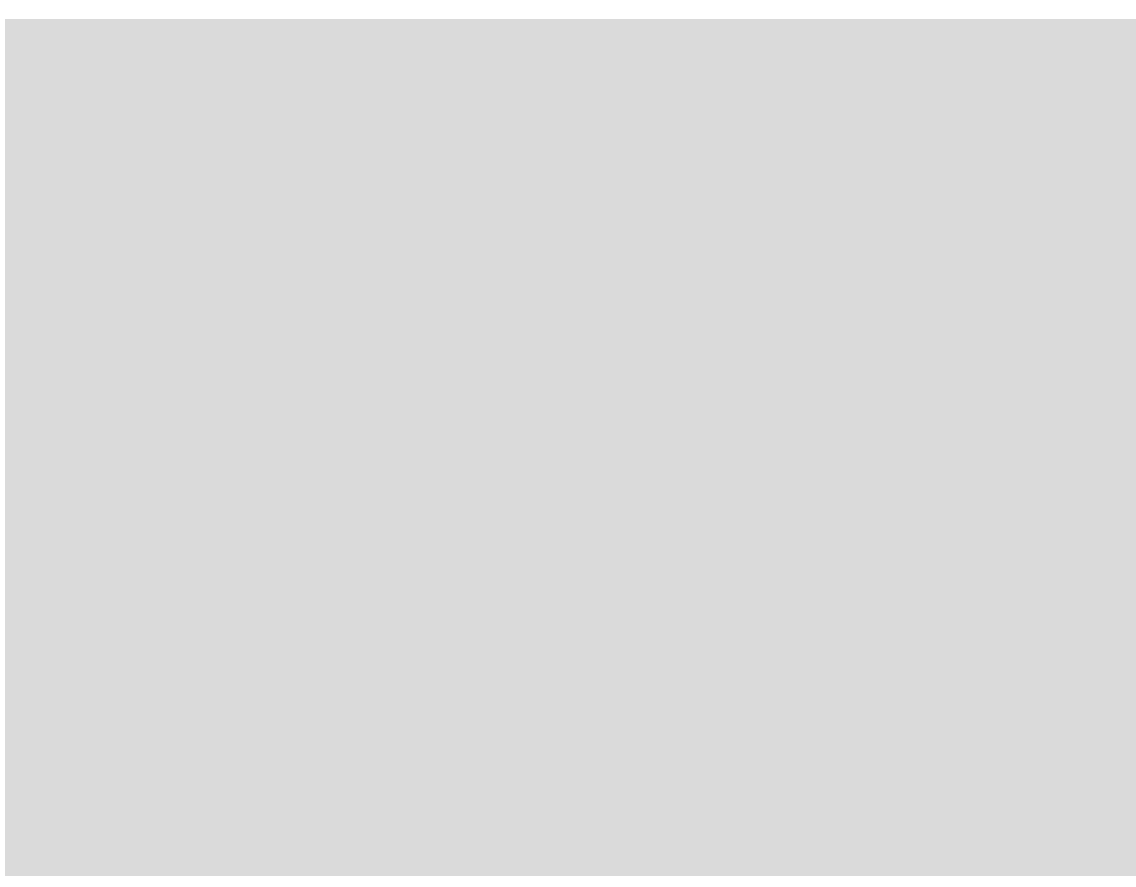

Abb. 2. Carl Julius Senff, Ansicht von Hohnstein in der Sächsischen Schweiz. Bleistift 3. Sept. 1829. Estnisches Kunstmuseum.

1830 bewilligte Summe von 200 Dukaten wurde ihm am 1. Februar 1830 an seine Berliner Adresse „Louisen Badt No 7 bey Madame Luckwald [...] remittiert“.27

Zwar sind wir über keine Einzelheiten von Senffs Aufenthalt in Berlin unterrichtet, doch dürfte von einer Begegnung mit dem bedeutendsten Baumeister dieser Stadt, mit Karl Friedrich Schinkel (1781-1840), auszugehen sein. Ins Jahr 1829 reichen denn auch die ältesten datierten Zeichnungen Senffs aus dessen überlieferten Konvolut zurück, anhand deren sich zum Teil seine Reiseroute und Aufenthaltsorte zurückverfolgen lassen. Es handelt sich um mehrere Bleistiftzeichnungen, die in zarter Strichführung Motive aus der Gegend um Dresden und der Sächsischen Schweiz zeigen. Nach ihrer Datierung sind sie alle im September 1829 entstanden. Das älteste Blatt datiert vom 1. September und zeigt das Wasserschloss Pillnitz vom Zschachwitzer Elbufer aus. ${ }^{28}$

27 EAA, 402-2-23084, Bl. 36.

28 Vgl. C. J. Senff, Pillnitz. Bleistift, bez.: Pillnitz d 1 Sept 1829. Estnisches Kunstmuseum [Eesti Kunstimuuseum, EKM], Inv.-Nr. G 963.

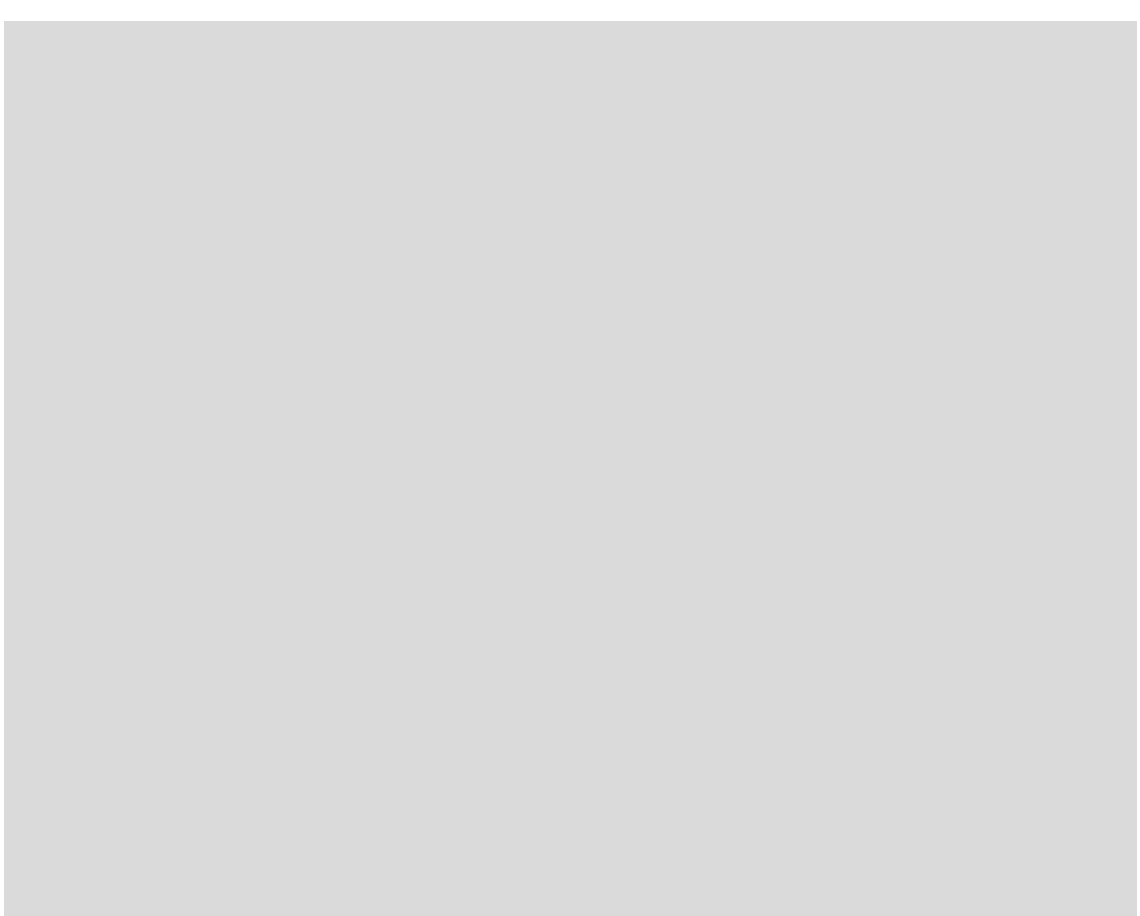

Abb. 3. Carl Julius Senff, Landschaft mit Gewässer. Bleistift, Sepia, 1828-1829. Estnisches Kunstmuseum.

Zwei Tage später, am 3. September, zeichnete er bereits in Hohnstein, im östlichen Elbsandsteingebirge, das er fälschlich mit „Hochstein“ bezeichnete (Abb. 2). Vom darauf folgenden Tag existiert eine Zeichnung von den Felspartien bei Brand aus dem tiefen Grunde ${ }^{29}$, dem von Hohnstein nur drei Kilometer entfernten, berühmten Felsplateau in der Sächsischen Schweiz. Von hier aus führte ihn die Route weiter zum Ausfluss der Polenz in die Elbe bei Schandau ${ }^{30}$, den er am 5. September besuchte, ehe mit Ansichten vom Königstein und Lilienstein ${ }^{31}$ sowie von Lohmen ${ }^{32}$ wo er am Monatsende zeichnete - seine Wanderungen durch die Sächsische

29 Vgl. C. J. Senff, Der Brand. Bleistift, bez.: der Brand aus dem tiefen Grunde. d 4 Sept 1829. EKM, Inv.-Nr. G 945 .

30 Vgl. C. J. Senff, Ausfluss der Polenz in die Elbe bei Schandau. Bleistift, bez.: Ausflus (sic!) d. Polenz in die Elbe bei Schandau d 5t Sept 1829. EKM, Inv.-Nr. G 955.

31 Vgl. C. J. Senff, Königstein und Lilienstein. Bleistift, 1829, bez.: Königstein u Lilienstein EKM, Inv.-Nr. G 967.

32 Vgl. C. J. Senff, Lohmen. Bleistift, bez.: Lohmen (31.IX, 1829! sic!). EKM, Inv.-Nr. G 953. 


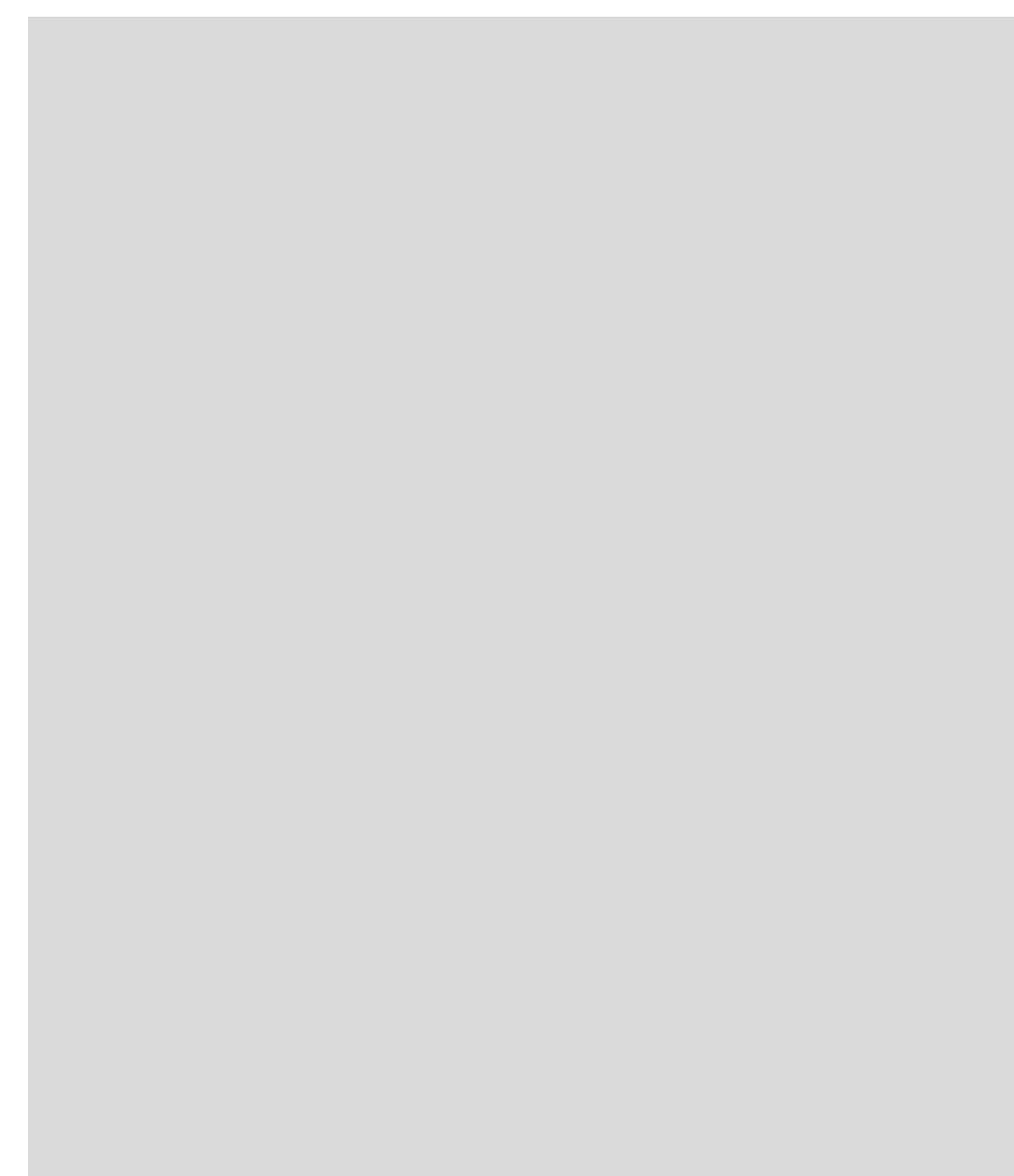

Abb. 4. Carl Julius Senff, Der Heinrichsturm in Prag. Aquarell. Estnisches Kunstmuseum.

Schweiz ihren Abschluss fanden. All diese Bleistiftskizzen verstehen sich als präzise Veduten von touristisch attraktiven Landschaftsszenen, die Carl Julius Senff mit feinfühligem Realismus in zarter, sparsamer Linienführung als Erinnerung festhielt. Sie stehen im Kontrast zu einigen erhalten gebliebenen Sepien (Abb. 3) ${ }^{33}$, die in ihrem weich fließenden Zeichenstil und der überhöhenden, idyllische Bildsprache noch stark an die empfindsam-klassizistische Landschaftskunst des Vaters erinnern, so dass zu vermuten ist, dass diese nicht lokalisierbaren Landschaftsszenerien noch aus der Zeit der Schulung unter der Ägide des Zeichenmeisters Carl August Senff - also vor Julius' Aufbruch ins Ausland - stammen.

\section{DIE REISEN NACH EUROPA}

Ob sich diesem Ausflug in Sächsische im Herbst 1829 sogleich die Weiterreise nach Böhmen anschloss oder ob er erst einmal wieder nach Berlin zurückkehrte, lässt sich weder aus den Akten, noch aus den Zeichnungen bestimmen, denn alle Ansichten aus Böhmen sind nicht datiert. Denkbar ist, dass er zunächst noch Nordböhmen besuchte, wo er mit Ansichten von Tetschen (Děčín) ${ }^{34}$ und Teplitz (Teplice) $)^{35}$ ähnliche Bleistiftzeichnungen hinterließ, wie wir sie von seinen sächsischen Wanderungen her kennen. Sein Aufenthalt in Prag und Wien ist wohl eher für das Jahr 1830 - nach dem Empfang seines Jahresstipendiums in Berlin und der Promotion in Jena - anzunehmen, da diese Darstellungen nun nicht mehr den relativ flüchtigen Skizzencharakter von Reisestudien in sicht tragen, sondern auf fein ausgearbeiteten Aquarellen (Abb. 4) und Sepien (Abb. 5) beruhen, die das Ziel präziser architektonischer Studien verfolgten. Diese Studien stehen im Zusammenhang mit der Serie von zehn Illustrationen in Aquatinta, die 1831 in Berlin unter dem Titel Die Domkirche zu St. Veit in Prag (vgl. Abb. 1) erschienen. Zwischen dem 6. und 15. Oktober 1830 datieren dann zwei Bleistiftzeichnungen und eine Sepia, die Motive aus Klosterneuburg bei Wien ${ }^{36}$ und vom Stift Melk ${ }^{37}$ in Niederösterreich sowie von der oberösterreichischen Landeshauptstadt Linz ${ }^{38}$ zeigen. Aus der Datierung wird ablesbar, dass Senff von Wien aus donauaufwärts seine Route wählte, so dass er vor oder nach dieser Wanderung durch Österreich seinen Aufenthalt in Prag angeschlossen haben könnte. Im Jahr 1830 wollte C. J. Senff nach

34 Vgl. C. J. Senff, Tetschen. Bleistift, 1829-1830, bez: Tetschen, aus dem Fenster des Gasthofes zur Sächsischen. EKM, Inv.-Nr. G 965.

35 Vgl. C. J. Senff, Teplitz. Bleistift, 1829-1830, bez.: Tetschen. EKM, Inv.-Nr. G 968

36 Vgl. C. J. Senff, Klosterneuburg. Bleistift, 1830, bez.: d. 6 Oct Klosterneuburg. EKM, Inv.-Nr. G 962

37 Vgl. C. J. Senff, Stift Melk. Bleistift, bez.: Kloster Melk d 11 Oct 1830. EKM, Inv.-Nr. G 951

38 Vgl. C. J. Senff, Linz. Bleistift, bez.: Linz d. 15 Oct 1830. EKM, Inv.-Nr. G 971. 


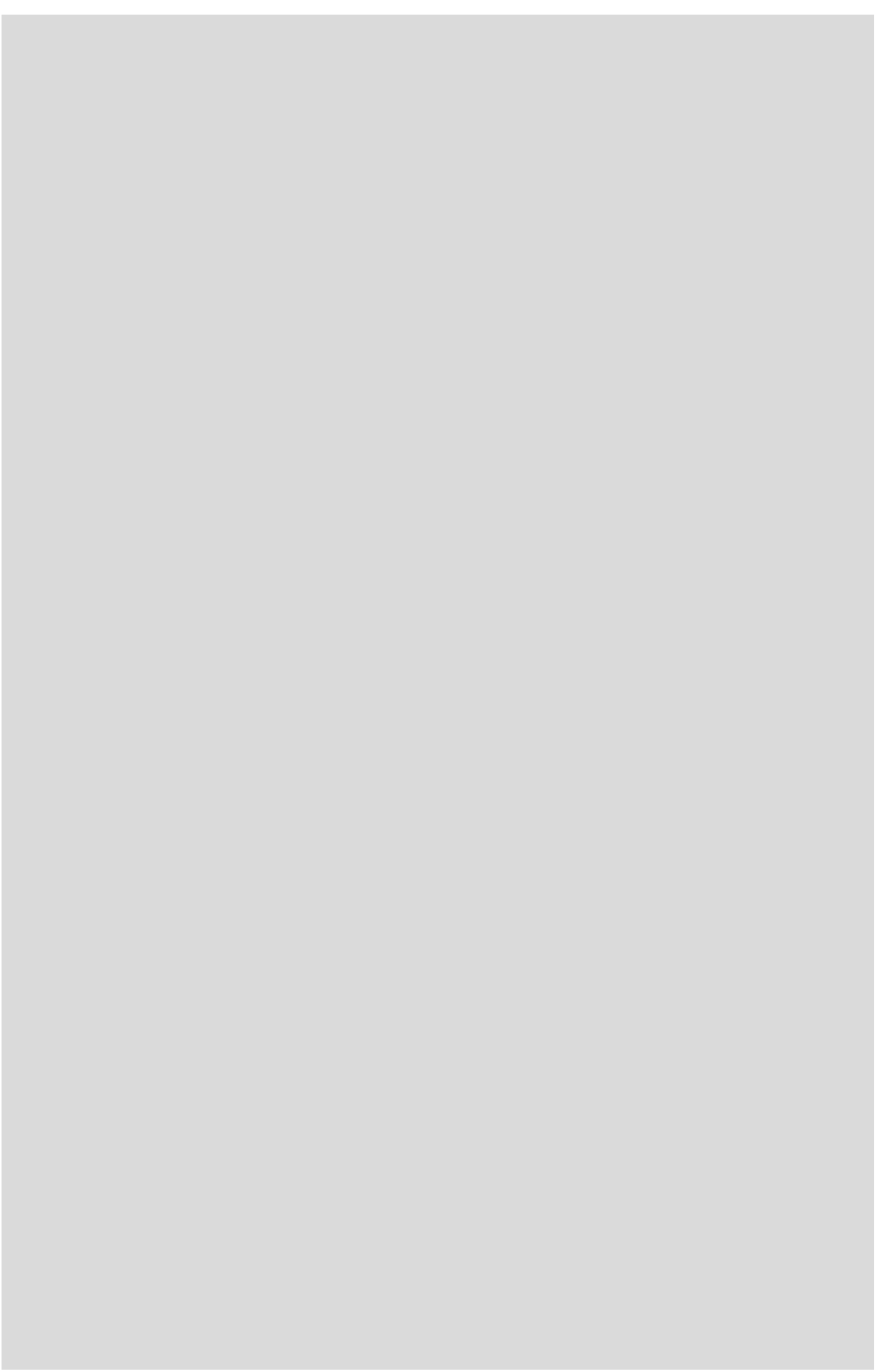

Abb. 5. Carl Julius Senff, Kirchenportal (Augustinerkirche Wien). Bleistift, Sepia, 1830 Estnisches Kunstmuseum.
Paris reisen ${ }^{39}$, jedoch war er wegen des Ausbruches der Julirevolution daran gehindert worden: „Ew. Magnifizenz werden sich erinnern, daß mein ältester Sohn, als er seine Reise ins Ausland auf kaiserliche Kosten antrat, um sich für das Fach der schönen Baukunst und der angewandten Mathematik auszubilden, von Einem Hochverordneten Conseil der hiesigen Universität die Instruktion erhielt, zur Erreichung dieses Zweckes ein Jahr in Berlin und München ein Jahr in Paris und ein Jahr in Italien vorzüglich in Rom sich aufzuhalten. Schon im August des vorigen Jahres wollte er nach Paris reisen, wo bekanntlich für angewandte Mathematik mehr gethan und geleistet wird, als an irgend einem anderen Orte, allein der rußische Gesandten in München verweigerte ihm einen Paß nach Paris wegen der daselbst ausgebrochenen Unruhen zu geben, und rieth ihm einige Zeit länger in München zu verweilen und abzuwarten, ob sich nicht bald die politischen Angelegenheiten so gestalten würden, daß seiner Reise nach Paris kein Hinderniß im Wege wäre. Da er vergeblich einige Zeit in München gewartet hatte, und ein längerer Aufenthalt daselbst nur verlorene Zeit für ihn gewesen wäre, so entschloß er sich nach Rom zu reisen, obgleich es ihm für den Zweck / seiner Reise vortheilhafter gewesen wäre, vorher nach Paris zu gehen, und sich daselbst wo möglich länger als ein Jahr aufzuhalten, aber als dann erst die Reise nach Rom angetreten. Jetzt sind von dem ihm zur Reise bestimmten Jahren schon zwei Jahre und dre Monate verstrichen und der russische Gesandte in Rom will ihm auch noch jetzt nicht eher seinen Paß nach Paris visieren, bis mein Sohn die specielle Erlaubniß höheren Orts vorher dazu aus gereicht hätte; ich ersuche deshalb Ew Magnifizenz gehorsamst, die vom russischen Gesandten in Rom verlangte specielle Erlaubniß zum Reisen nach Paris sobald als möglich auszuwirken weil mein Sohn sonst nur zur Hälfte den wissenschaftlichen Zweck seiner Reise erreichen wird, und in der Folge die ihm obliegenden und zu übernehmenden Pflichten nicht genügend erfüllen könnte. Dorpat den 5 Sept 1831.“40

Die Wartezeit auf die Reiserlaubnis nach Frankreich muss Carl Julius unerträglich lang erschienen sein. Über seinen Aufenthalt in München ist nicht mehr bekannt als diese Tatsache des Wartens auf die Erledigung seiner Passformalitäten, die seitens der russischen Behörden nicht geregelt wurden, weil Russland ein Überspringen

39 Vgl. EAA, 402-2-23084, Bl. 40 recto.

40 Ibidem, Bl. 39 recto und verso. 
des revolutionären Funkens auf das Zarenreich befürchtete. Auch ein Gesuch des Rektors an das Ministerium in St. Petersburg konnte nicht bewirken, dass ihm die Erlaubnis zu dieser Reise gegeben wurde. ${ }^{41}$ Mit einer Wanderung ins benachbarte Landshut, wo er am 13. April 1831 Studien zu gotischen Architekturdetails an der dortigen Stiftsbasilika St. Martin betrieb ${ }^{42}$, glaubte er sich wenigstens dem Zweck seines Studienaufenthaltes in Deutschland zu nähern. Ein eigenhändiger Reisebericht des Stipendiaten aus Rom an den Rektor der Universität Dorpat gibt uns dann schließlich ein authentisches Zeugnis vom weiteren Verlauf der Auslandsreise des Canditaten C. J. Senff:

„Ew. Magnifizens / Werden zu Anfange dieses Jahres meinen letzten Bericht aus München erhalten haben. Im April ging ich nach Regensburg und Nürnberg, um die vielen würdigen Denkmale altdeutscher Kunst, die diese beiden Orte enthalten, zu studieren. Die Mauern schöner und reicher Gebäude, die ich hier fand, feßelte mich bis Ende Mai an diesen Orten. Gern hätte ich früher schon meine Reise nach Paris angetreten; allein dahin wurde mir der Paß verweigert. Da ich schon zu Anfang des Frühjahrs nach Italien gehen wollte, gestatteten es ebenfalls die dortigen politischen Unruhen nicht. Ich glaubte dafür meine Zeit nicht beßer anwenden zu können als zu jener kleinen Reise:

$\mathrm{Zu}$ Anfange des Juni erhielt ich endlich von unserem Gesandten die Erlaubniß nach Italien gehen zu dürfen und ich eilte dafür so schnell als möglich durch Tyrol bis Trient. Hier hielt ich mich einige Tage auf um die Domkirche ein zwar kleines aber dafür schönes Denkmal byzantinischen Styls kennen zu lernen, und ging dann nach Verona, welches mehrere Reste aus römischer Zeit und weiter Kirchen im schönen Style des achten Jahrhunderts enthält, und sich die Kirchenarchitectur von den herkömmlichen Formen des römischen Styls lossagte und immer eignen Charakter annehme, der zwar nicht so viel Sorgfalt und Schönheit in der Ausführung einzelner Ornamente darbietet als der ältere römische Styl, dagegen aber eine durchdachtere und consequentere Constructionsweiße und schönere Verhältniße der ganzen Maßen. Ich hielt mich dafür 10 Tage hier auf

41 EAA, 402-2-23084, Bl. 40 recto und verso.

42 C. J. Senff, Gotische Architekturdetails. Bleistift, bez.: Landshuth d. 13 April 1831. EKM, Inv.-Nr. G 941

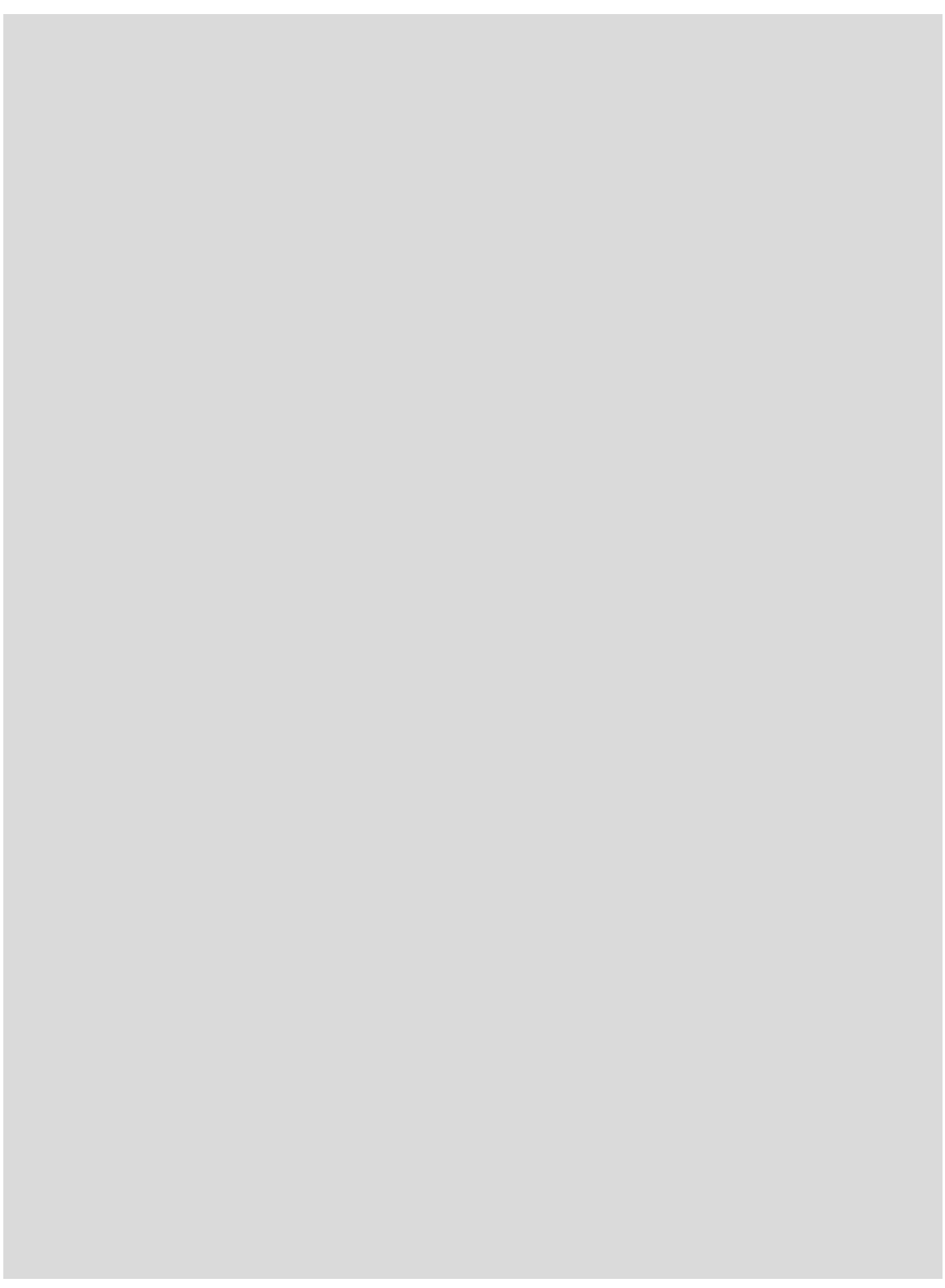

Abb. 6. Carl Julius Senff, Piazza dei Signori in Verona. Bleistift, 1831. Estnisches Kunstmuseum.

um diese Gebäude mit Aufmerksamkeit durchzusehen und Einiges davon zu zeichnen [(Abb. 6)].

Von Verona ging ich nach Venedig: Ich hielt es nicht für nothwendig mich in Vincenza aufzuhalten, obgleich hier mehr und schöne 
Gebäude des großen Palladio sind, denn dieser Styl ist der in welchem die jetzigen Architekten ihre erste Schule machen, und daher sind die meißten dieser Gebäude / sehr bekannt, übrigens wußte ich, dass ich deren wieder in Venedig finden würde. - Venedig weicht mir in Lebensweise und Sitten, so auch in seiner Architektur von allen anderen Städten bedeutend ab, daher es für den Architekten stets eine lehrreiche Schule seyn wird. Nicht die berühmte Marcuskirche, die größten Palaste (sic!) und Tempel allein sind es, die hier den Architekten beschäftigen und zum Studium auffordern; jedes kleine Privatgebäude hat etwas so Fremdartiges so romantisch Schönes daß man hier für Jahre Beschäftigung und Studium fände. Als ich 3 Wochen hier geblieben war machte mich die stets wachsende Sonnenglut so bald als möglich nach Rom zu gehen, ich reiste also über Padua und Ferrara wo ich weniger Aufforderung zu bleiben fand, bis Bologna. So originell diese Arkadenstadt ist so enthält sie doch wenige Gebäude die einer strengen Aufmerksamkeit werth sind; einige Gebäude aus dem 15t Jahrhundert sind fast das Einzige, daher ich mich beeilte nach Florenz zu gehen. Ein früheres Studium unserer Werke, die über die toskanische Architektur erschienen sind, erleichterten mir es sehr in kurzer Zeit die zahlreichen Meisterwerke dieser herrlichen Stadt zu durchmustern. Nur wenige mir noch nicht bekannte Kunstwerke zeichnete ich und besuchte fleißig die Gemäldegalerien und Kunstsammlungen.

Nach meinem Aufenthalt von 14 Tagen ging ich über Siena nach Rom wo ich in den letzten Tagen des Juli anlangte: Fast 4 Wochen brauchte ich um die Sehenswürdigkeiten dieser unendlich reichen Stadt flüchtig anzusehen, welches ich nöthig fand, um später mit Ruhe und Besonnenheit die Gegenstände meines Studiums auszuwählen. Im Museo des Vaticans zeichnete ich mir die meisten Reste antiker Ornamente ab, die ich nicht wie die übrigen Monumente Roms in den besten Kupferwerken finden kann. Diese Ornamente waren mir besonders wichtig da sie in einer ziemlich vollständigen Weise den Uebergang aus dem älteren römischen Style in den des $8 \mathrm{t}$ Jahrhunderts nachweisen welcher sich dann an die verschiedenen mittelalterlichen Style bis zur vollendetsten gothischen Baukunst anschließt. Um mich von dem vielen Sehen und Copieren zu erholen, arbeitete ich einige Projecte in den Baustylen für welche ich jetzt das Material gesammelt hatte, aus, und hatte die Freude daß sie den Beifall mehrer ausgezeichneter Künstler fanden.
Indeßen war der September fast vergangen und da ich noch hoffte im Dezember nach Frankreich gehen zu dürfen beeilte ich mich jetzt, nach Neapel zu gehen. Auf dem Wege besah ich das prachtvolle Kloster / Monte Cassino, die antiken Reste bei Capua und das Schloß Caserta. Neapel selbst enthielt mehrere recht schätzbare Gebäude des Mittelalters und viele gute Privathäuser neuester Zeit. Vor allem aber feßelte mich lange das unendlich reiche Museum in welchem fast alles zusammengestellt, was die Nachgrabungen in Herkulaneum und Pompeji Pästum Starba (sic!) und anderen Orten geliefert haben Die Maße der Gegenstände ist so groß, daß man schon zum Besehen lange Zeit braucht, wie viel mehr wenn man einiges zeichnet. Viel Zeit kostet es ebenfalls die paradiesischen Umgegenden Neapels wenn auch nur flüchtig zu besehen; ich machte die erste kleine Reise über Sorent (sic!) auf Capri ${ }^{43}$ wo mehrere Ruinen aus der Zeit Tibero (sic!) zu sehen sind, ging dann über Amalfi nach Salern (sic!), beide Orte enthalten schöne Kirchen aus dem Mittelalter. Von hier ging ich nach Pästum (sic!) um die würdigen Ruinen griechischer Zeit zu sehen, und kehrte dann nach Pompeji zurück, wo ich mich zehn Tage aufhielt um mich mit dem freundlichen Style der alten Privatgebäude vertraut zu machen. Eine zweite Tour führte mich längs der Küste von Pozuoli (sic!) Bajä (sic!) bis zum Capo Missenae wo Ruinen um Ruinen gedrängt das ganze Ufer einnehmen.

Nachdem ich 2 Monate auf diesem fast lehrreichsten Theil der Reise in Italien verwendet hatte, kehrte ich nach Rom zurück, wo ich die Nachricht vorfand daß meine Bitte um Erlaubniß nach Frankreich gehen zu dürfen, abgeschlagen sey. Ich werde daher den Winter hier bleiben, und so viel als möglich von den hiesigen Kunstanstalten und vom Umgange mit ausgezeichneten Künstlern Nutzen zu ziehen suchen, da hier eine gute Bibliothek fehlt in welcher ich mich mit dem theoretischen Theile meines Faches beschäftigen könnte. Sobald als die Jahreszeit erlaubt will ich noch einige Orte im Gebirge besuchen, an welchen ich gute mittelalterliche Gebäude finde; und gegen das Ende Februar meine Rückreise über Pisa Genua Mayland, langs dem Rheine hinunter und so weit antreten, um im Juli in der Heimath freudig und getrost die Verpflichtung zu übernehmen die man mir anvertrauen wird.

43 C. J. Senff, Die Blaue Grotte auf Capri. Bleistift, Gouache, 1831, bez.: Grotta Pagano azura (Capri). EKM, Inv.-Nr. G 947. 
Mit der Versicherung, daß ich endlich that was in meinen Kräften stand, und durch eine dauerhafte Gesundheit unterstützt, auch wärend (sic!) des halben Jahres das mir zur Reise übrig ist, alle Anstrengung nur auf die Verfolgung des vorgesteckten Zieles nehmen werde, / bitte ich Ew. Magnifizens mir Ihre Wohlgesonnenheit und Ihr Zutrauen zu erhalten. Hochachtungsvoll /Ew Magnifizens /gehorsamster C. J. Senff / Rom d. 9ten Dec./27te Nov. 1831“.44

$\mathrm{Zu}$ den Werken, die C. J. Senff während der Zeit des hier wiedergegeben Reiseberichts geschaffen hatte, gehören sicher mehrere Darstellungen aus den Alpen ${ }^{45}$, eine antike Ruine ${ }^{46}$ sowie die Ansicht des Hafens von Desenzano del Garda vom 13. Juni $1831^{47}$ u. a. m. Es ist bekannt, dass er in Rom mit seinem Onkel Adolf Senff (1785-1863), dem Raffaele di fiori, zusammentraf, der ihn als Mitbegründer und Vorstandsmitglied des Römischen Kunstvereins den Weg zu der in der Ewigen Stadt etablierten Künstlerrepublik ebnete und mit dem er einige Wanderungen unternahm. ${ }^{48}$ Doch weder die Hoffnung, seine Studienreise mit einem Besuch von Paris abzuschließen, noch die designierte Stelle eines Professors der Baukunst in Tartu antreten zu können, erfüllten sich, denn die letzten Blätter der Akte Carl Julius Senff berichten von dessen traurigem Schicksal, dass sich bereits erfüllen sollte, noch ehe die Knospe des jungen Talents zu voller Blüte gereift war: „Ew Excellenz muß ich zu meinem u. der hiesigen Univ. großtem (sic!) Leidwesen gehorsamst berichten, daß der auf Allerhöchsten Befehl ins Ausland zur weiteren wissenschaftlichen Ausbildung gesandte Herr Cand. phil. Julius Senff, Sohn des Herrn Prof. extraord. Hofrath Senff hieselbst, am 19. April a. o. St. in Mailand an einem bösartigen Nervenfieber nach einem 14tägigen Krankenlager, ohnerachtet der häuslichen und ärztlichen Pflege gestorbnen ist". ${ }^{49}$

44 EAA, 402-2-23084, Bl. 43 und Bl. 44.

45 C. J. Senff, Wasserfall. Sepia. EKM, Inv.-Nr. G 992; C. J. Senff, Gebirgslandschaft mit Tal. Sepia, 1830-1831. EKM, Inv.-Nr. G 993.

46 C. J. Senff, Tempel der Minerva Medica bei Rom. Sepia, 1831. EKM, Inv.-Nr. G 979.

47 C. J. Senff, Desenzano del Garda. Bleistift, bez.: Hafen in Defezano 13. Juni 1831. EKM, Inv.-Nr. G 960

48 Vgl. Adolf Senff. Malerei und Zeichnungen. Ausstellung aus Anlaß seines 200. Geburtstages Andreas Hüneke, Adolf Senff (Leipzig: Seemann, 1986); Bärbel Kovalevski, Adolf Senff 17851863 (Berlin: Bärbel Kovalevski, 2009).

49 EAA, 402-2-23084, Bl. 46; vgl. auch B1. 45

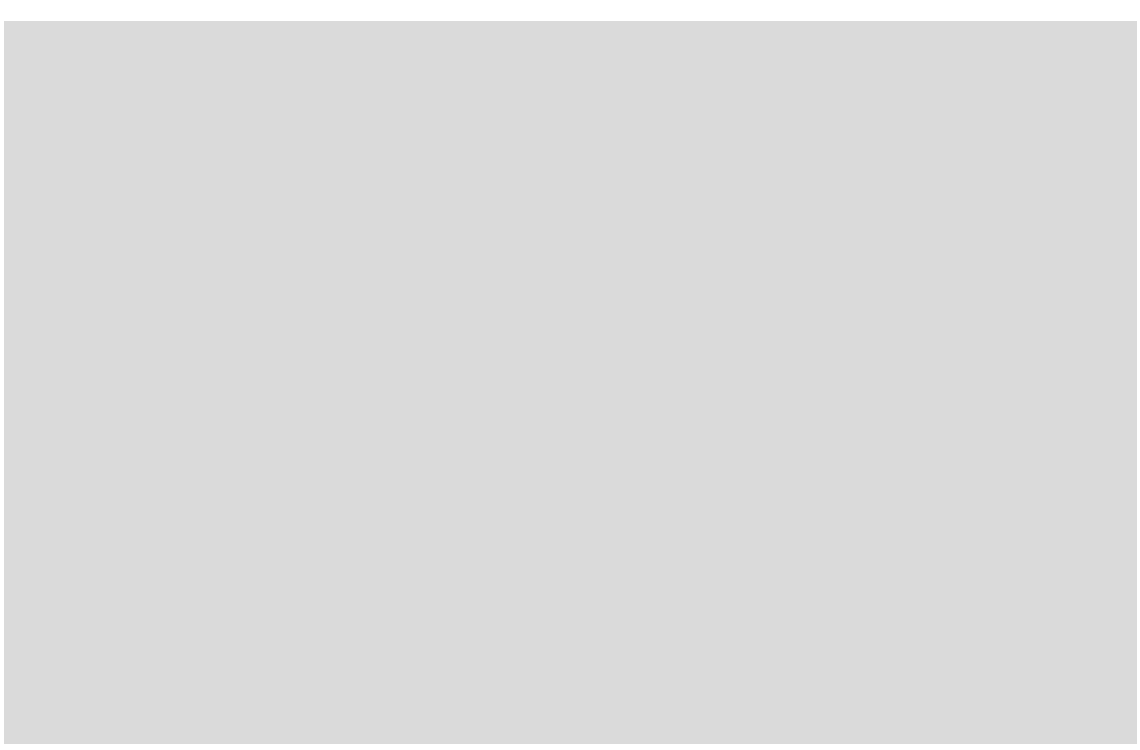

Abb. 7. Karoline Schilling, geb. Senff, Zwei Wickenblüten. Gouache, Febr. 1827. Bibliothek der Universität Tartu.

Auch seine älteren Schwester Karoline (1801-1840), die sich 1827 mit Georg Gustav Schilling, vermählte und die ebenfalls unter der Hand ihres Vaters zur Künstlerin herangebildet worden war, blieb nur wenig Zeit, sich als Blumenmalerin zu entfalten, da sie bereits am 20. Mai 1840 im Alter von 39 Jahren verstarb und sechs Kinder hinterließ.$^{50}$ Von ihrem kleinen Euvre hat sich so gut wie nichts erhalten. So bezeugt wenigstens eine kleine Gouache mit der Darstellung von Zwei Wickenblüten (Abb. 7), die sie im Jahre ihrer Vermählung dem mit dem Familie Senff befreundeten Professor der Baukunst, Johann Wilhelm Krause, widmete, von ihren ausgezeichneten Talenten, die sich freilich gemäß der damaligen gesellschaftlichen Traditionen bei einer Künstlerin ganz auf den engen Bereich der Blumenmalerei zu begrenzen hatte.

Carl Julius Senffs Aktivitäten bildeten einen bemerkenswerten Teil der Brücke für den gemeinsamen deutsch-estnischen Kulturraum und sein unerwarteter Tod in Mailand am 19. April 1831 beeinflusste sowohl seinen Vater Karl August Senff stark als dass er auch eine ungefüllte Lücke im deutschbaltischen Kulturerbe hinterließ.

50 Vgl. Carl Theodor Hermann, „Erinnerungen. I und II“, Baltische Monatsschrift, Bd. XXXVIII, hrsg. von Robert Weiss (Reval, 1891), 92; Neumann, Lexikon baltischer Künstler, 137. 
Gerd-Helge Vogel: Carl Julius Senff (1804-1832) - A Short Artist's Life As An Architect, Draughtsman And Graphic Artist

Keywords: Carl Julius Senff; Italian Trips; Alma Mater Tartuensis; Baltic Art And Architecture

\section{SUMMARY}

Carl Julius Senff was a talented artist with wide-ranging knowledge of art and architecture, who first received art lessons from his father Carl August Senff in Tartu. From 1823 to 1827, Carl Julius Senff studied philosophy at the University of Tartu and illustrated several publications while still a student. For example, in 1825 he published a textbook on perspective called Perspective für Landschaftsmaler zum Selbststudium.

Although Carl Julius Senff's life was cut short, he was still able to travel extensively. In 1829, he travelled to Jena for three years where he acquired a doctoral degree in philosophy. His earliest drawings also date from this period and, based on them, we can also partially reconstruct the route of his travels. From Jena, he travelled to Prague, Vienna and Munich. In 1831, he travelled to Italy where he lived in Rome. The young artist and scholar was destined to become the new professor of architecture at the University of Tartu but, unfortunately, Carl Julius Senff fell ill during his last trip and died in Milan.

The article is based to a great degree on unpublished archival documents as well as the personal correspondence of Carl Julius Senff and his father Carl August Senff.

\section{CV}

Gerd-Helge Vogel (b. 1951), Assistant Professor (lecturer) Emeritus at the Zurich University of Applied Arts (ZHdK), Switzerland, and Greifswald University, Germany. In 1999 and 2002, he was Assistant Professor / Lecturer at the Estonian Academy of Arts, Tallinn. The organiser of many conferences on the Romantic movement and curator of numerous exhibitions in Germany, Switzerland, and Poland, he has also published extensively on the art of the Enlightenment, the Romantic movement, history of gardening, scientific illustrations and poster art. 